\title{
Avaliação da Eficácia de um Programa de Orientação Vocacional no $9^{\circ}$ ano Evaluation of the Effectiveness of a Vocational Counseling Program of 9th year
}

\author{
Diana Rocha, Ana Rodrigues Costa, Ana Rita Cardoso \\ Universidade Fernando Pessoa
}

\begin{abstract}
Resumo
A Orientação Vocacional é um processo que decorre ao longo do ciclo vital. O objetivo geral deste trabalho consiste em elaborar e implementar um programa de orientação vocacional, "Projetando o meu futuro", dentro de um plano de estudo que pretende avaliar a eficácia do mesmo. Os participantes $(\mathrm{N}=114)$ foram divididos em grupo experimental $(\mathrm{N}=54)$ e em grupo de controlo $(\mathrm{N}=60)$. Ambos os grupos foram avaliados com a Escala de Avaliação da Tomada de Decisão Vocacional (pré e pós-teste) que demonstrou ter boas qualidades psicométricas. O programa elaborado revelou-se eficaz na promoção da capacidade de tomada de decisão vocacional. Palavras-chave: desenvolvimento para a carreira; orientação vocacional; tomada de decisão vocacional; adolescência.
\end{abstract}

\begin{abstract}
Vocational Counseling is a process that happens throughout the life cycle. The main objective is to develop and implement a vocational counseling program, "Designing My Future", within a study plan to assess its effectiveness. The participants $(\mathrm{N}=114)$ were divided into experimental group $(\mathrm{N}=54)$ and control group $(\mathrm{N}=60)$. Both were evaluated with the Vocational Decision-Making Assessment Scale (pre and post-test) which showed good psychometric qualities. The developed program proved to be effective in promoting vocational decision-making capacity.

Keywords: career development; vocational counseling; vocational decision-making; adolescence.
\end{abstract}

Pelo prisma vocacional, a adolescência é caracterizada como a fase de transição entre a fantasia e o realismo (Super 1995; Taveira, 1999). As escolhas e os sonhos profissionais passam a ser formulados com base na apreciação das atividades que realizam e na autoavaliação de interesses por certas disciplinas.

Nesta fase, apesar de expressarem as suas preferências vocacionais, os adolescentes não se sentem confiantes com as mesmas, apresentando muitas incertezas no que concerne ao seu papel vocacional, pois ainda não exploraram suficientemente o seu eu face ao mundo escolar e profissional (Taveira, 1999).

É na adolescência que o indivíduo recapitula o conjunto de identificações infantis com o intuito de as absorver segundo uma nova configuração da identidade (Roque \& Santos, 2004). O estabelecimento de uma preferência profissional pode representar uma oportunidade de reconstrução de referências que permitam evitar a confusão de identidade (Guichard, \& Huteau, 2002).

Os processos de exploração e de tomada de decisão assumem uma especial importância no desenrolar do desenvolvimento vocacional na adolescência (Blustein, 1997; Kracke, 2002; Porfeli \& Skorikov, 2010; Taveira, 2000; Van Esbroek, Tibos, \& Zaman, 2005). Assim há que enfatizar o processo de exploração vocacional e a sua relação com o processo de formação da identidade e da motivação por um lado e as suas implicações a nível da tomada de decisão por outro (Blustein, 1997; Flum \& Blustein, 2000).

A intervenção vocacional encontra-se inerente a qualquer esforço do indivíduo com a intenção de melhorar o seu conhecimento sobre o desenvolvimento da carreira e assim melhorar as suas decisões (Spokane, 2004).

Neste sentido, recomenda-se o desenho de intervenções psicológicas que promovam o desenvolvimento de conhecimentos, atitudes e competências de gestão e desenvolvimento da carreira ao longo da vida, como os programas de educação para a carreira, que vão para além das intervenções mais pontuais, destinadas a ajudar as pessoas a decidir o que fazer da sua vida ocupacional num futuro próximo (Carvalho, Pocinho, \& Silva, 2010; Königstedt \& Taveira, 2010). Os programas de desenvolvimento de carreira têm ainda um papel importante no que concerne à prevenção do insucesso e do abandono escolar (Pocinho, 2011).

Com os programas de educação para a carreira pretende-se criar as condições fundamentais para que cada indivíduo possa adquirir um conjunto de competências essenciais como, por exemplo, competências académicas básicas e competências para tomar decisões de carreira, bem como adquirir conhecimento acerca de si próprio e das oportunidades educativas e ocupacionais existentes (Pocinho, 2011).

No contexto escolar, caberá aos psicólogos dos Serviços de Psicologia e Orientação (SPO), em colaboração com os professores e outros agentes educativos, a dinamização de diversas modalidades de intervenção vocacional, desde a consulta psicológica individual aos programas de orientação, passando pelas atividades de informação e de aproximação ao contexto de trabalho (Gamboa, Paixão, \& Jesus, 2011). 
Autores como Arrington (2000) e Taveira (2000) são da opinião que os conteúdos e objetivos inerentes aos programas de educação para a carreira devem fomentar a exploração do self, do mundo do trabalho e do desempenho de papéis de vida, de forma a criar condições ótimas para a tomada de decisão vocacional realista, ou seja, a exploração vocacional permite o aumento do conhecimento acerca do Eu e do mundo, promove a construção de uma visão mais diferenciada da realidade e incrementa o grau de consciência do sujeito acerca das suas intenções e ações.

Em Portugal existem, há mais de duas décadas, programas estruturados de orientação de carreira e, desde a última década, programas de desenvolvimento vocacional inspirados na perspetiva da educação para a carreira. A maioria destes tem sido alvo de avaliação e resulta da colaboração entre investigadores e psicólogos a trabalhar no terreno (Araújo, Taveira, \& Lemos, 2004; Coelho, Correia, \& Taveira, 2004; Taveira, 2002).

O presente estudo tem como principais objetivos: elaborar, implementar e avaliar a eficácia de um programa de orientação vocacional, em grupo, administrado a alunos do $9 .^{\circ}$ ano de escolaridade.

\section{Método}

Participantes. Os participantes $(\mathrm{N}=114)$, com idades compreendidas entre 14 e 15 anos, foram divididos em dois grupos: grupo experimental $(\mathrm{N}=54)$ e grupo de controlo $(\mathrm{N}=60)$.

Materiais. Tendo em conta os objetivos deste estudo, foi utilizado, o questionário sociodemográfico como instrumento de recolha de dados relativamente à identificação do participante, assim como à caracterização dos seus microssistemas e antecedentes escolares.

A Escala de Avaliação da Tomada de Decisão Vocacional (Rocha \& Costa, 2016), tem como principal objetivo aferir o modo como as pessoas pensam acerca do seu processo de tomada de decisão. Pela análise descritiva dos itens constata-se a existência de uma distribuição normal, sendo que os coeficientes de assimetria e de curtose situam-se abaixo da unidade, apoiando a sensibilidade da mesma (Guéguen, 1999). Relativamente à sua consistência interna, o Alpha de Cronbach assume um valor de 0,753 refletindo um grau aceitável de uniformidade. Da análise fatorial revela-se a presença de 7 dimensões, ainda que as dimensões IV, V, VI tenham só 2 fatores e a VII apresente apenas 1 . No entanto, todos eles manifestam um coeficiente de saturação elevado, superior a 0,50 , espelhando a validade do instrumento.

O programa de Orientação Vocacional "Projetando o Meu Futuro" foi construido com base na integração de diversos projetos de intervenção, nomeadamente, programas de orientação vocacional destinados aos alunos do 9..$^{\circ}$ ano de escolaridade (Calado, 2009; Moura, Sampaio, Gemelli, Rodrigues, \& Menezes, 2005), Ser Activo - Explorar para Decidir (Konigstedt, 2008; 2010), ProjetOrienta 9..$^{\circ}$ ano (Costa, 2015), Futuro Bué! (Faria, Araújo, Taveira, \& Pinto, 2008).
Este pretende contribuir para o sucesso de uma tomada de decisão vocacional consciente. Neste sentido pretende estimular o envolvimento consciente dos alunos no seu processo de exploração vocacional, aperfeiçoar as estratégias associadas ao processo de tomada de decisão, promover a capacidade de exploração pessoal, fomentando o processo de autoconhecimento, e aumentar o conhecimento dos alunos sobre as variâncias de cursos e profissões existentes.

Desta forma, o mesmo foi fragmentado em seis categorias de intervenção, a saber: Apresentação do programa, Projeto Vocacional, Exploração do "Eu", O "Eu" na Exploração do Contexto Profissional, O "Eu" na Exploração da Decisão e Conclusão.

Foi ainda elaborada a autorização para a Direção do Agrupamento e para os Encarregados de Educação, relativamente à participação dos educandos no Programa, bem como a declaração de consentimento informado e as fichas de avaliação de cada sessão e do programa em geral.

Procedimento. Com a aprovação do projeto de investigação, por parte da Comissão de Ética da Universidade Fernando Pessoa e posterior aprovação do agrupamento de escolas para a implementação do programa de Orientação Vocacional, iniciou-se a recolha da amostra, na medida em que foram abertas as inscrições de participação. Seguidamente, emitiu-se a todos os encarregados de educação, o convite para a participação numa reunião onde foi apresentado o programa de orientação vocacional e solicitada a autorização de participação no programa e a assinatura do consentimento informado para a participação no estudo de investigação.

Salienta-se que foram, devidamente, assegurados o direito de anonimato e confidencialidade da amostra, dado que cada elemento da mesma foi identificado, em todos os documentos, através de um código.

A administração do programa "Projetando o Meu Futuro" decorreu ao longo do $3 .^{\circ}$ período, iniciando-se em abril e terminando em junho, com a duração de 9 sessões, ressalvando-se que a primeira e a nona sessão se destinaram aos encarregados de educação, tendo uma periocidade semanal, com a duração de 01:30h. Contou ainda com sessões individuais previamente agendadas a pedido dos alunos.

Para analisar o impacto da intervenção torna-se fundamental a existência de, pelo menos, dois momentos de avaliação, ou seja, um pré-teste e um pós-teste face à administração do programa (Almeida \& Freire, 2008). $\mathrm{Na}$ prática, culminou na administração da Escala de Avaliação da Tomada de Decisão Vocacional, desenvolvida por Rocha e Costa (2016). Ainda com o objetivo de avaliar a eficácia do programa, no final de todas as sessões, assim como no final do programa, os alunos eram convidados a preencher um questionário de avaliação.

\section{Resultados}

Avaliação da Eficácia do Programa. Com o intuito de verificar a existência de diferenças entre o GE e o GC 
antes e após a administração do programa de intervenção realizou-se o teste $t$ para amostra independentes.

Pela análise da tabela 1, conclui-se que, em média, os indivíduos do GC apresentam maior capacidade de tomada de decisão vocacional do que os indivíduos do GE. Considerando a análise da variância, podemos afirmar que não existem diferenças significativas entre os indivíduos do GE e os indivíduos do GC ao nível da tomada de decisão vocacional $(t(111)=0,301, p=0,766)$, no pré-teste. Assim, face ao momento de pré-teste, os indivíduos do GC não relatam maior capacidade de tomada de decisão vocacional do que os indivíduos do GE.

Tabela 1.

Diferenças na Capacidade de Tomada de Decisão Vocacional entre o GE e o GC, antes da Administração do Programa

\begin{tabular}{cccccc}
\hline & & $M(D P)$ & $G L$ & $t$ & $P$ \\
\hline Pré & GE & $65,48(5,824)$ & 111 & $-0,301$ & $0,766^{*}$ \\
teste & GC & $65,85(7,218)$ & & & \\
\hline Nota. *p & $<0,05$ & & & &
\end{tabular}

A partir dos dados da tabela 2 podemos concluir que, em média, os indivíduos do GE evidenciam maior capacidade de tomada de decisão vocacional do que os indivíduos do GC. Considerando a análise da variância, podemos afirmar que existem diferenças significativas entre os indivíduos do GE e os do GC ao nível da capacidade de tomada de decisão vocacional $(t$ $(112)=3,687, p=0,000)$, a favor do primeiro grupo. Face ao momento de pós-teste os indivíduos do GE relatam maior capacidade de tomada de decisão vocacional do que os indivíduos do GC.

Tabela 2.

Diferenças na Capacidade de Tomada de Decisão Vocacional entre o GE e o GC, após a Administração do Programa

\begin{tabular}{cccccc}
\hline & & $M(D P)$ & $G L$ & $t$ & $P$ \\
\hline Pós & GE & $73,04(7,945)$ & 112 & 3,687 & $0,000^{*}$ \\
teste & GC & $67,95(6,783)$ & & & \\
\hline Nota. ${ }^{*} p<0,05$ & & & &
\end{tabular}

No final da implementação do programa solicitou-se o preenchimento de um questionário de avaliação global do mesmo. Nesse questionário, cada indivíduo classificava a sua avaliação sobre uma escala de "Mau", "Insuficiente", "Suficiente", "Bom" e "Muito Bom".

Perante a sua análise conclui-se que 34 dos participantes $(63 \%)$ classificam o programa como "Muito Bom", seguindo-se a classificação de "Bom" atribuída por 18 participantes $(33,3 \%)$ e a classificação de "Suficiente" atribuída por 2 participantes $(3,7 \%)$.

\section{Discussão}

O propósito da elaboração e implementação do programa depreende-se com o importante papel que os mesmos apresentam na criação de condições favoráveis à adquisição de um conjunto de competências essenciais, por parte do indivíduo, a destacar: competências académicas básicas e competências para a tomada da decisão vocacional realista, assim como adquirir conhecimento acerca de si próprio, das oportunidades educativas e ocupacionais existentes, do mundo do trabalho e do desempenho de papéis de vida (Arrington, 2000; Pocinho, 2011; Taveira, 2000). Estes ainda se mostram significantes no que concerne à prevenção do insucesso e do abandono escolar (Pocinho, 2011).

A avaliação da eficácia do programa, na tomada de decisão vocacional, envolveu dois grupos, como já referimos: um submetido à intervenção com base no programa, que constituiu o GE e outro sem intervenção, que constituiu o GC. Tanto o GE como o GC foram submetidos à avaliação da capacidade de tomada de decisão vocacional, através da Escala de Avaliação da Tomada de Decisão Vocacional (Rocha \& Rodrigues da Costa, 2016), em dois momentos distintos, nos mesmos tempos experimentais.

Avaliamos estatisticamente as diferenças entre os dois grupos, nos momentos antes e após a administração do programa. Desta forma, após a análise dos resultados, é possível concluir que se atingiu, positivamente, o objetivo relativo à eficácia do programa.

Ao compararmos os indivíduos que foram submetidos ao programa de intervenção (GE) com os indivíduos que não foram submetidos (GC) verificamos uma evolução estatisticamente significativa no primeiro grupo de indivíduos.

No primeiro momento de avaliação (pré-teste), comparando os grupos denotamos que os indivíduos do GC, apesar de apresentarem uma média ligeiramente acima face ao GE, a diferença não se constitui como estatisticamente significativa em relação à capacidade de tomada de decisão vocacional. No segundo momento de avaliação (pós-teste), ainda numa comparação entre os dois grupos ressalva-se, em média, maior evolução dos indivíduos do GE, relativamente à capacidade de tomada de decisão vocacional, verificando-se diferenças estatisticamente significativas.

Assim, estes resultados são reforçados pela literatura e vão ao encontro ao defendido por Araújo, Taveira e Lemos (2004), Arrington (2000), Calado (2009), Moura, et al (2005), Coelho, Correia e Taveira (2004), Königstedtn e Taveira (2010), Pinto (2002), Pocinho (2011) e Taveira (2000; 2002), sendo que os mesmos defendem que a implementação deste tipo de intervenções parece promover o desenvolvimento de conhecimentos, atitudes e competências de gestão e desenvolvimento da carreira ao longo da vida. Especificamente, possibilitam a criação de condições fundamentais para fomentar a exploração do self, do mundo do trabalho e do desempenho de papéis de vida, de forma a criar condições ótimas para a tomada de decisão vocacional realista.

No entanto ressalva-se ainda, que o processo de tomada de decisão não se encontra somente dependente da intervenção vocacional, no contexto da implementação de programas de educação na carreira. Um conjunto de traços de personalidade e comportamentos dos indivíduos justificam a execução ou não de objetivos vocacionais (Jome \& Phillips, 2005; Young et al., 2007). 
A avaliação do programa por parte dos seus intervenientes constitui um fator essencial para a aferição da sua eficácia, dado que esta depende do grau de envolvimento do indivíduo no processo de intervenção (Moura et al, 2005). Através da análise do questionário de avaliação do programa, que era anonimo, foi possível verificar que, na sua maioria, os participantes avaliaram a intervenção em "Muito Bom". Este facto vem atestar a eficácia da implementação do programa, assim como a pertinência das atividades desenvolvidas.

Face ao exposto compreende-se a importante relevância da orientação vocacional, nomeadamente dos programas de educação para a carreira, associada ao processo de tomada de decisão, ao nível da exploração pessoal e da promoção do bem-estar na adolescência.

\section{Referências}

Araújo, A., Taveira, M. C., \& Lemos, M.S. (2004). Uma experiência de intervenção precoce no desenvolvimento vocacional em contexto pré-escolar. In M. C. Taveira., H. Coelho, H. Oliveira, \& J. Leonardo (Orgs.), Desenvolvimento vocacional ao longo da vida: Fundamentos, princípios e orientações (pp. 197-209). Coimbra: Livraria Almedina.

Arrington, K. (2000). Middle Grades Career Planning Programs. Journal of Career Development. $2^{\circ} 27,103$ 109. doi/10.1177/089484530002700204

Blustein, D. (1997). A contexto-rich perspective of career exploration across the life roles. The Career Development Quarterly. 45, 260-274. http://eds.a.ebscohost.com/eds/pdfviewer/pdfviewer?i $\mathrm{d}=1 \&$ sid=b20f9520-eeff-4bc9-

bebd4d8b89eb2e94\%40sessionmgr4010\&hid=4102

Calado, I. (2009). Um programa de planeamento da carreira para estudantes do $9^{\circ}$ ano de escolaridade. Tese de Mestrado, Faculdade de Psicologia e Ciências da educação, Universidade de Lisboa, Lisboa, Portugal. http://repositorio.ul.pt/bitstream/10451/3068/1/ulsd_t m_Ines_Calado.pdf

Carvalho, R., Pocinho, M., \& Silva, C. (2010). Comportamento adaptativo e perspectivação do futuro: Algumas evidências nos contextos da educação e da saúde. Psicologia: Reflexão e Crítica. 23, 411-418. http://digituma.uma.pt/bitstream/10400.13/192/1/Com portamentoadaptativo.pdf

Carvalho, M., \& Taveira, M. (2012). A implementação de decisões vocacionais: Revisão da literatura. Revista Brasileira de Orientação Profissional. 1º 13 , 27-3. http://pepsic.bvsalud.org/scielo.php?script=sci_arttext \&pid=S1679-33902012000100005

Coelho, H., Correia, L., \& Taveira, M. C. (2004). Programas de desenvolvimento vocacional na Casa Pia de Lisboa. In M. C. Taveira, H. Coelho, H. Oliveira, \& J. Leonardo (Coords.), Desenvolvimento vocacional ao longo da vida: Fundamentos, princípios e orientações (pp. 260-267). Coimbra: Livraria Almedina.

Flum, H., \& Blustein, D. L. (2000). Reinvigorating the study of vocational exploration: a framework for research. Journal of Vocational Behavior. 56, 380-404. doi:10.1006/jvbe.2000.1721
Gamboa, V., Paixão, M., \& Jesus, S. (2011). A eficácia de uma intervenção de carreira para a exploração vocacional. Revista Brasileira de Orientação Profissional. $\quad 2^{\circ} 12, \quad 153-164$. http://pepsic.bvsalud.org/scielo.php?script=sci_issues \&pid=1679-3390\&lng=pt\&nrm=iso

Gati, I. \& Tal, S. (2008). Decision-making models and career guidance. In J. Athanasou, \& R. Esbroeck (Eds.), International handbook of career guidance (pp. 157-185). Springer Netherlands. http://link.springer.com/chapter/10.1007\%2F978-14020-6230-8_8

Germeijs, V., \& Verschueren, K. (2007). High school students' career decision-making process: Consequences for choice implementation in higher education. Journal of Vocational Behavior. 70, 223241.

Guichard, J. (2001). A century of career education: Review and perspectives. International Journal for Educational and Vocational Guidance. 1, 155-176.

Guichard, J., \& Huteau, M. (2002). Psicologia da orientação. Lisboa: Instituto Piaget

Jome, L., \& Phillips, S. (2005). Counseling for choice implementation. In S. Brown \& R. Lent (Eds.), Career development and counseling: Putting theory and research to work (pp. 466-481). London: John Wiley and Sons.

Königstedt, M. (2008). Educação e carreira: Estudo de avaliação da eficácia de uma intervenção psicológica. Tese de Mestrado, Instituto de Educação e Psicologia, Universidade do Minho, Braga, Portugal. http://repositorium.sdum.uminho.pt/bitstream/1822/83 14/1/TESE.final.revista.pdf

Königstedt, M., \& Taveira, M. (2010). Exploração vocacional em adolescentes: avaliação de uma intervenção em classe. Paidéia. 47º20, 303-312. http://www.scielo.br/pdf/paideia/v20n47/a03v20n47.p df

Kracke, B. (2002). The role of personality, parents and peers in adolescents career exploration. Journal of Adolescence. 25, 19-30. doi: 10.1006/jado.2001.0446

Mortimer, J., Zimmer-Gembeck, M. J., Holmes, M., \& Shanahan, M. (2002). The process of occupational decision making: Patterns during the transition to adulthood. Journal of Vocational Behavior. 61, 439465. http://ac.els-cdn.com/S0001879102918853/1s2.0-S0001879102918853-main.pdf?_tid=7477c700$66 \mathrm{e} 8-11 \mathrm{e} 7-9 \mathrm{f} 4 \mathrm{c}-$ 00000aab0f6c\&acdnat=1499853603_8d09016d4388f 61eb68f73dedbea09c9

Munhoz, I., \& Melo-Silva, L. (2011). Educação para a Carreira: Concepções, desenvolvimento e possibilidades no contexto brasileiro. Revista Brasileira de Orientação Profissional. $1^{\circ} 12$, 37-48. http://pepsic.bvsalud.org/scielo.php?script=sci_arttext \&pid=S1679-33902011000100006

Papalia, D., Olds, S., \& Feldman, R. (2010). Desenvolvimento Humano. (10 ${ }^{\mathrm{a}}$ ed., C. Vercesi, et al, Trad.). Porto Alegre: AMGH Editora.

Phillips, S. D., Blustein, D. L., Jobin-Davis, K., \& White, S. F. (2002). Preparation for the school-to-work transition: The views of high school students. Journal 
of Vocational Behavior. 61, 202-216. https://www.researchgate.net/publication/222079066_ Preparation_for_the_School-to-

Work_Transition_The_Views_of_High_School_Stud ents

Pinto, H., Taveira, M., \& Fernandes, M. (2003). Os professores e o desenvolvimento vocacional dos estudantes. Revista Portuguesa de Educação. $1^{\circ} 16,37$ 58. https://www.researchgate.net/publication/26465020_ Os_professores_e_o_desenvolvimento_vocacional_do s_estudantes

Pocinho, M. (2011). Avaliação de um programa de educação para a carreira no ensino fundamental. Revista Brasileira de Orientação Profissional. $2^{\circ} 12$, 253-265.

http://pepsic.bvsalud.org/scielo.php?script=sci_arttext \&pid=S1679-33902011000200012

Pocinho, M., \& Correia, A. (2009). Causalidade (locus de controle) das dificuldades de tomada de decisão em alunos do ensino médio: Estudos de validação. Trabalho apresentado na Conferência Internacional de Avaliação Psicológica: Formas e Contextos.

Pocinho, M. D., Correia, A., Carvalho, R. G., \& Silva, C. (2010). Género, família e orientação vocacional. Revista Brasileira de Orientação Profissional. $2^{\circ} 11$, 201-212.

<http://pepsic.bvsalud.org/scielo.php?script=sci_artte xt\&pid=S1679$33902010000200005 \& \operatorname{lng}=$ pt\&nrm=iso >

Porfeli, E. J., \& Skorikov, V. B. (2010). Specific and diverse career exploration during late adolescence. Journal of Career Assessment. $1^{\circ} 18$, 46-58. doi:10.1177/1069072709340528

Roque, H., \& Santos, P. (2004). Decisão vocacional e desenvolvimento da identidade. In C. Machado, L. Almeida, M. Gonçalves, \& V. Ramalho (Org.), Avaliação psicológica: Formas e contextos (pp. 120135). Braga: Psiquilíbrios Edições

Skorikov, V. (2007). Continuity in adolescent career preparation and its effects on adjustment. Journal of Vocational Behavior. 1\%70, 8-24.

Spokane, A. R. (2004). Avaliação das intervenções de carreira. In L. Leitão (Ed.), Avaliação psicológica em orientação escolar e profissional (pp. 455-473). Coimbra: Quarteto.

Super, D. (1995). Valeus: their nature, assessment, and practical use. In D. Super, B. Sverko, \& C. Super (Eds.), Life roles, values, and careers (pp. 54-61). San Francisco: Jossey-Bass Publishers.

Taveira, M. (1999). Intervenção precoce no desenvolvimento vocacional. Psicologia: Teoria, investigação e prática. $1^{\circ} 4$, 173-190. http://repositorium.sdum.uminho.pt/bitstream/1822/34 753/1/Interven\%C3\%A7\%C3\%A3o\%20precoce\%20n o\%20desenvolvimento\%20vocacional.pdf

Taveira, M. (2000). Exploração e desenvolvimento vocacional de jovens: Estudo sobre as relações ente a exploração, a identidade e a indecisão vocacional. Tese de Doutoramento, Instituto de Educação e Psicologia, Universidade do Minho, Braga, Portugal. http://repositorium.sdum.uminho.pt/handle/1822/1985 6

Taveira, M. C. (2002). Educação e desenvolvimento de carreira ao longo da vida. A intervenção vocacional baseada em programas. In Homenagem ao Professor Doutor J. Ferreira Marques. Universidade de Lisboa, Faculdade de Psicologia e de Ciências da Educação. Sacavém: Stória Editores.

Van Esbroeck, R., Tibos, K. \& Zaman, M. 2005). A dynamic model of career choice development. International. Journal for Educational and Vocational Guidance. 5, 5-18. doi:10.1007/s10775-005-2122-7

Whiston, S., \& Keller, B. (2004). Expanding research concerning family influences on career development: Cultivating a number of brown spots. The Counseling Psychologist. 32, 612-617. doi: 10.1177/0011000004266009

Young, R. A., Marshall, S. K., Domene, J. F., Graham, M., Logan, C., Templeton, L., et al. (2007). Meaningful actions and motivated projects in the transitions to adulthood: Two case illustrations. International Journal of Educational and Vocational Guidance. 7, 149-158. https://link.springer.com/article/10.1007\%2Fs10775007-9127-3 